\title{
Design and Implementation of Narrative Map Based on Hand Scroll -- An Example of Zheng He's voyage to the West
}

\author{
Jie Shen ${ }^{\text {a, b, c, }}$ Lufei Zhao ${ }^{\text {a, c }}$, Nina Sun ${ }^{\text {d, *, Tengfei Chai }}{ }^{\text {a, c }}$ \\ ${ }^{a}$ Key Laboratory of Virtual Geographic Environment (Nanjing Normal University), Ministry of Education, Nanjing 210023, China; \\ shenjie@njnu.edu.cn(J.S),zlf1412015111@outlook.com(L.Z),201302152@njnu.edu.cn (T.C) \\ ${ }^{b}$ Jiangsu Center for Collaborative Innovation in Geographical Information Resource Development and Application, Nanjing 210023, \\ China \\ c School of Geography, Nanjing Normal University, Nanjing 210023, China \\ ' Academy of Fine Arts, Jiangsu Second Normal University,210013, China; 2399872982@qq.com \\ * Corresponding author
}

Keywords: hand scroll, narrative map, map design, Zheng He's nautical chart

\begin{abstract}
:
Cartographic theory before the mid-1850s was structured on science, art and technology, during that period established the basic theory and technology of cartography. Along with the debate continuing, art and cartographic theory gradually divided, and in recent years, a variety of cultural and creative products for the geographic information industry have emerged, setting off a boom in the creative application of art and cartography. The narrative map is a cross-disciplinary product of cartography, culture, art and design, and it has attracted much attention in recent years. The narration was first used to describe the causes and consequences of events and then gradually enriched to form narratology which was studied in the temporal dimension and lacked knowledge of the spatial dimension. At the end of the 20th century, narratology began to take a "spatial turn", and narrative cartography was born.
\end{abstract}

Narrative map integrates the expressive advantages of narratology in the temporal dimension and cartography in the spatial dimension, visually expressing the development of stories in time and space and having a certain cultural bearing function. Most of the existing maps are creatively designed to show the geographic environment or the humanities with the freehand drawing technology, rarely integrating the actual geographical map with the artistic expression of the form. Hand scroll painting, as one of the forms of traditional Chinese painting, is the best carrier of Chinese painting narrative, its viewing style has a unique and interesting interactive character that brings sensory stimulation and connects the viewer more closely to the picture, while the moving perspective point of view matches the flowing characteristics of the narration in time or space.

Therefore, the main content of this study is to apply the narrative form of hand scroll to the expression of the narrative map, incorporating the concept and technique of creative design to visualize the main content of the story.

As the largest series of maritime expeditions in world history before the European geographic discoveries at the end of the 15th century, Zheng He's great voyage is worth remembering. The original Zheng He's Nautical Chart is not directional and the existing repainted version does not have a strong sense of art and immersion, while the sequence of Zheng He's voyage in the chart, unfolding from right to left, is very suitable for the artistic expression of the spatial and temporal characteristics of the narrative map in the form of hand scroll. Therefore, we take Zheng He's voyage to the West as the narrative object and design the narrative space, element and the spatial temporal process of the voyage, was expressed taking hand scroll as the narrative carrier. Following methods were designed for achieving the above targets:

1) The selection of narrative elements based on narrative space, we should collect identified places and events on each route through research and analysis of narrative objects, then select the important expression events in the story space and map them to the geographical space according to the type.

2) In order to visualize the ancient voyage, the geographic base map is designed according to the style of Chinese ancient map based on the Arts and Crafts style, while overlaying a hill shading topographic map for display. The Landmark symbols are expressed through hand-drawn illustrations in a realistic style of customary illustration with colors fitting the base map. 
3) Map layout design referring the structure of hand scroll, traditional hand scrolls are composed of several parts such as BaoShou, preface, core, etc (Figure 1). The painted Chinese paintings are framed on core, the three parts, title label, preface and preface and postscript, need to be handwritten. So the layout of the map should be designed according to the structural characteristics of the hand scroll and the framing material, the paper map follows the traditional decoration process, where the core is drawn by computer, while the web map is drawn by computer according to the needs of the hand scroll structure.

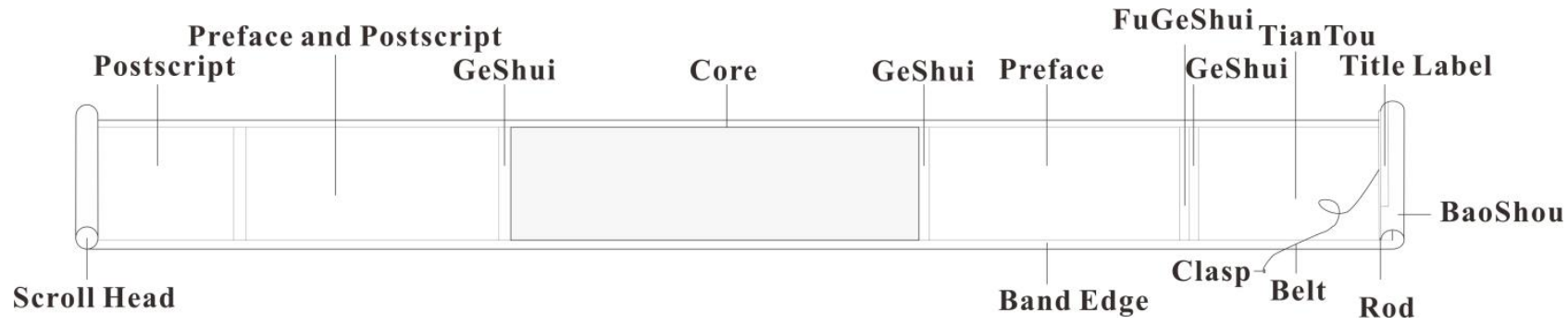

Figure 1. Hand scroll decoration structure drawing.

4) Dynamic interactive design of web map, the webpage interface is designed with dynamic effects according to the way of hand scroll opening, and the different dynamic routes are displayed according to the spatial temporal sequence of Zhenghe's Voyage, to avoid the obscuration of the overall route display by expressed events, narrative elements are displayed through an interactive pop-up design.

Through the integration of innovative design in this way, the map achieves better readability and vivid visual effect. The dynamic interactive map in the form of the scroll on the website can accommodate more narrative plots and dynamic vessels guide the visual focus to meet more interest needs. The expression form in narrative map design can promote the cross-border integration of map and culture, deepen the cultural connotation of the map and enrich the expression of the achievements of the geographic information industry.

\section{Acknowledgements}

This research was funded by Research on Visual Map Creative Products Based on VR/AR Technology (2021RD11) 